This item was submitted to Loughborough's Research Repository by the author.

Items in Figshare are protected by copyright, with all rights reserved, unless otherwise indicated.

\title{
Hydroxypropyl methylcellulose as a novel tool for isothermal solution crystallization of micronized paracetamol
}

PLEASE CITE THE PUBLISHED VERSION

http://dx.doi.org/10.1021/cg4009637

PUBLISHER

(C) 2014 American Chemical Society

VERSION

AM (Accepted Manuscript)

\section{PUBLISHER STATEMENT}

This work is made available according to the conditions of the Creative Commons Attribution-NonCommercialNoDerivatives 4.0 International (CC BY-NC-ND 4.0) licence. Full details of this licence are available at: https://creativecommons.org/licenses/by-nc-nd/4.0/

\section{LICENCE}

CC BY-NC-ND 4.0

\section{REPOSITORY RECORD}

Reis, Nuno M., Zizheng K. Liu, Cassilda Reis, and Malcolm R. Mackley. 2015. "Hydroxypropyl Methylcellulose as a Novel Tool for Isothermal Solution Crystallization of Micronized Paracetamol". figshare. https://hdl.handle.net/2134/18358. 


\title{
HPMC as a novel tool for isothermal solution crystallisation of micronized Paracetamol
}

\author{
Nuno M Reis ${ }^{\mathrm{a}, \mathrm{b},{ }^{*} \text {, Zizheng K Liu }}{ }^{\mathrm{a}}$, Cassilda M Reis ${ }^{\mathrm{a}}$, and Malcolm R Mackley ${ }^{\mathrm{a}}$ \\ ${ }^{a}$ Department of Chemical Engineering and Biotechnology, University of Cambridge, New Museums \\ Site, Pembroke Street, Cambridge CB2 3RA, UK \\ ${ }^{b}$ Department of Chemical Engineering, Loughborough University, Loughborough LE11 3TU, UK
}

${ }^{*}$ Corresponding author: Phone +44 (0) 1509222 505; Fax +44 (0) 1509223 923; e-mail n.m.reis@lboro.ac.uk 


\title{
HPMC as a novel tool for isothermal solution crystallisation of micronized Paracetamol
}

\author{
Nuno M Reis ${ }^{\mathrm{a}, \mathrm{b}, \dagger}$, Zizheng K Liu ${ }^{\mathrm{a}}$, Cassilda M Reis ${ }^{\mathrm{a}}$, and Malcolm R Mackley ${ }^{\mathrm{a}}$ \\ ${ }^{a}$ Department of Chemical Engineering and Biotechnology, University of Cambridge, New Museums \\ Site, Pembroke Street, Cambridge CB2 3RA, UK \\ ${ }^{b}$ Department of Chemical Engineering, Loughborough University, Loughborough LE11 3TU, UK
}

\begin{abstract}
Pulmonary inhalation is increasingly being selected as a preferred route for the delivery of both small and large drug macromolecules for the treatment of a range of pathologies. The direct crystallisation of micronized powders, in particular Paracetamol remains difficult, as it requires the ability to work in high solution supersaturations where agglomeration, wall crusting and heterogeneous nucleation hinders the control of crystal size and crystal size distribution. Polymer additives are recognised to help driving the production of a given polymorph or controlling crystal shape by means of adsorption on crystal surface. With the aim of exploiting the polymer-control nucleation and growth of crystals for enhanced direct crystallisation of micronized powders, batch cooling crystallisation of Paracetamol in water was carried out in the presence of $0.1-0.8 \% \mathrm{w} / \mathrm{w}$ hydroxypropyl methylcellulose (HPMC). In the presence of polymer the onset of nucleation was delayed and extended beyond the cooling time of the solution, resulting in an isothermal cooling crystallisation and the production of micronized Paracetamol with a mean crystal size $D_{50}$, in the range of $15-20 \mu \mathrm{m}$ and an improved crystal size distribution. Equally, the rate generation of solution cloudiness was reduced by over 3-fold for the highest HPMC concentration tested, with no detectable impact on final product yield. The mechanisms for nucleation delay and growth inhibition by HPMC is unknown, however a modification of crystal's shape observed upon the addition of HPMC to the solution suggested it might be related to mass transfer limitations and inter-molecular hydrogen bounding between the large HPMC and the small drug molecules. This technique can potentially be used for direct crystallisation of other micronized drugs.
\end{abstract}

Keywords: Paracetamol, micronized, cooling crystallisation, isothermal crystallisation, HPMC

\footnotetext{
${ }^{\dagger}$ Corresponding author: Phone +44 (0) 1509222 505; Fax +44 (0) 1509223 923; e-mail n.m.reis@lboro.ac.uk
} 


\section{Introduction}

Pulmonary inhalation is being increasingly selected as a preferred route for the delivery of both small and large drug macromolecules for the treatment of a range of pathologies. The fast expanding dry powder inhalers (DPI) market is expected to reach over US\$13bn per annum by 2016, benefiting from the remarkable development in drug formulation and inhalation device designs mainly in recent years. ${ }^{1}$ Dispatch such technological advancement, the production of micronized $(\sim 1-10 \mu \mathrm{m})$ active pharmaceutical ingredients (APIs) still relies on traditional milling and grinding techniques for size reduction of crystals. These methods apply high energy that result in a final product with broad size distribution, limited crystallinity and poor flowing properties with limited dispersability. ${ }^{2}$ An alternative technique that is in increasing adoption by industry is spray drying. ${ }^{3}$ It allows producing micronized APIs of desirable size however particles are amorphous and have a larger tendency to re-crystallise or degrade. ${ }^{2}$ It is therefore recognised that direct crystallisation of micronized crystals solution is advantageous, however producing small crystal sizes requires operation with the high supersaturations at which the control of agglomeration, wall crusting, crystal size and crystal size distribution (CSD) becomes extremely challenging. ${ }^{4}$

Crystallisation is an important process in the chemical, pharmaceutical, biotechnological and allied industries, as it is used extensively for separation and purification of organic fine chemicals or APIs and production of microsized APIs for drug delivery. ${ }^{5}$ Important product characteristics such as crystal size, CSD, and crystal morphology are determined by the operating conditions during the crystallization process, which include: supersaturation, temperature profiling, the presence of additives, air-water interface, anti-solvent addition rate, fluid mixing, residence time, materials of crystalliser, and agglomeration/breakage phenomena.

Stagnant crystallisation experiments have shown that polymers and other additives can help driving the production of a given polymorph, ${ }^{6-9}$ reducing the crystal size ${ }^{10}$ or controlling the crystal shape. ${ }^{9}$ The list of polymers tested is extensive and includes both hydrophilic and hydrophobic powders, such as ethylcellulose, methylcellulose (CM), polyethylene glycol (PEG), polyvinyl alcohol (PVA), agar, gelatine, polyvinyl pyrrolidone (PVP), poly(ethylene oxide) (PEO), corn starch, carrageenan, polyethylene (PE), polyvinyl chloride (PVC), polymethyl methacrylate (PMMA) and hydroxylpropyl methylcellulose (HPMC) to name a few. ${ }^{3}$,-12 Generally, crystallisation additives are effective in producing polymorphs of pharmaceuticals while avoiding others by encouraging the growth of a desirable form or disrupting the growth of the other form. ${ }^{8,9,13,14}$ Modification of crystal shape occurs as the polymer deposition on the crystal surface inhibits the growth of specific surfaces of the crystal. ${ }^{13}$ Reduction of particle size by means of long molecular weight additives was only marginally reported in the literature, and exception is perhaps the work of Femi-Oyewo and 
Spring ${ }^{10}$, although a recent work by Xie et al. ${ }^{12}$ showed that salbutamol sulphate crystal size was reduced to less than $10 \mu \mathrm{m}$ upon presence of polymer additives HPMC, polyvinylpyrrolidone (PVP K25), lecithin and Span 85 during anti-solvent water-ethanol crystallisation.

There is also very few studies touching the effect of organic polymers in the kinetics of crystallisation. An exception is the work of Raghavan et al. ${ }^{15}$ who have studied anti-solvent crystallisation of hydrocortisone acetate (HA) in the presence of four different polymers (HPMC, PVP, MC and PEG400). In the absence of solution additive, the nucleation of HA was observed to be spontaneous; however in the presence of a polymer the nucleation was delayed by several hours and a level of growth inhibition of HA was observed. The mechanism of nucleation retardation was explained by the authors in terms of association of HA with the polymer through hydrogen bonding whilst the growth inhibition was related to the adsorption of polymer to the surface of the crystal.

HPMC is a natural polymer extensively used in the pharmaceutical industry as a tableting ingredient and as a binder ${ }^{16}$ because of its regulatory approval status. Steckel et al. ${ }^{2}$ have shown that HPMC can act as a stabilising hydrocolloid during the production of micronized fluticasone-17-propionate by spray drying, resulting in one order of magnitude reduction in particle size.

In this work small concentrations of HPMC were used for manipulating the kinetics of cooling crystallisation of Paracetamol, which offered a new level of control over the nucleation and crystal growth under well mixed conditions and allow reduced crystal size and improved CSDs. The mean crystal size, CSD and crystal morphology have been experimentally evaluated for a concentration of HPMC in range of $0-0.8 \% \mathrm{w} / \mathrm{w}$ using standard powder characterisation techniques.

\section{Experimental section}

\section{Materials}

Paracetamol or acetaminophen (CAS number, 103-90-2, min 99\% purity) and HPMC (CAS 900465-3, typical MW = 10,000) were supplied by Sigma-Aldrich, Inc (Dorset, UK).

\section{Crystallisation procedure}

Paracetamol was crystallised in water by dissolving a given amount of Paracetamol at $70{ }^{\circ} \mathrm{C}$ corresponding to a saturated solution at $65^{\circ} \mathrm{C}$, and rapidly cooling it to room temperature $\left(20^{\circ} \mathrm{C}\right)$. For each experiment, 3.0 grams of Paracetamol were dissolved in 60 grams of deionised water in the presence of $0-0.8 \% \mathrm{w} / \mathrm{w}$ of HPMC (percentage of mass of HPMC per mass of water), giving a maximum drug to polymer ratio of $6.25: 1$ in weight or 413:1 in molar basis. The crystallisation vessel consisted of $42 \mathrm{~mm}$ internal diameter, $d$, glass-jacketed unbaffled tank with a liquid height, $h$ $=d$ corresponding to a working volume of $60 \mathrm{ml}$ (Figure 1). The solution was magnetically stirred 
at $70^{\circ} \mathrm{C}$ for 1 hour to ensure complete dissolution of Paracetamol, by circulating hot water (HAAK, Fisons DCS B3, Pacific Diagnostic, Inc. USA). The maximum working volume of the tank was 83 $\mathrm{ml}$, and all experiments were done in the presence of a free surface. Mixing was provided using an $18 \mathrm{~mm}$ length magnetic stirrer at $300 \mathrm{rpm}$, controlled by a stirrer plate (KIKA, Labortechnik, Janke \& Kunkel Gmbh \& Co KG. UK).

The Paracetamol solubility estimated from Granberg\&Rasmuson ${ }^{19}$ for working temperature of 65 ${ }^{\circ} \mathrm{C}$ and $0 \%$ HPMC was $\sim 48.3$ grams per kilogram of water, i.e. $\sim 2.9$ grams per $60 \mathrm{ml}$ of water.

The cooling crystallisation was started by quickly switching circulating hot water in a 3-way valve to chilled water at $20 \pm 0.1{ }^{\circ} \mathrm{C}$ from a refrigerated/cooling circulator (Grant LTD20, Cambridge, UK), which induced a rapid temperature quenching in the crystallisation vessel. The experiments were continued for about 45 minutes from the start of the cooling process, which was found sufficient to complete of crystals growth and fully deplete supersaturation in the solution. The supersaturation ratio of pure paracetamol solution defined as $S=C / C^{*}$ was estimated as equal to 3.8 based on the solubility data for pure paracetamol reported by Granberg\&Rasmuson. ${ }^{19}$ The initial paracetamol concentration remained constant for all HPCM concentrations tested.

The crystallisation vessel was equipped with a thermocouple (TME 2050, k-type) and a reflectance fibre optical probe for on-line monitoring of the temperature and turbidity of crystallisation solution, respectively (Figure 1). Details of the optical probe are given in section 2.3.

At the end of each crystallisation run, the slurry was vacuum filtered through a $0.2 \mu \mathrm{m}$ cut-off PTFE membrane (Whatman Inc, USA) and the crystals washed with $3 * 1.5 \mathrm{ml}$ of deionised water at room temperature. Paracetamol crystals were then dried in a desiccator for about 12 hours after which the dry weight was determined in an analytical balance. The crystal size and CSD were then characterised using an automated optical microscopy system, and crystal morphology analysed by Scanning Electron Microscopy (SEM). Crystal system was identified using powder X-ray diffraction (XRD) and the thermal behaviour using Differential Scanning Calorimetry (DSC) techniques.

\section{Monitoring of solution turbidity}

Solution cloudiness or turbidity was monitored real-time using a reflectance optical fibre probe. The optical probe (FCR 7UV200, Avantes, Eerbeek, Netherlands) consisted of $6 \times 200 \mu \mathrm{m}$ diameter optical fibres carrying the light from a deuterium halogen light source and a $7^{\text {th }}$ fibre in the core of the probe delivering the scattered light to a spectrometer connected to a PC, which was controlled by Avasoft (Avantes, Eerbeek, Netherlands). The probe was inserted though the lid of the crystalliser at a certain angle to avoid direct reflection from the walls of the crystalliser. The crystalliser was fully covered with aluminium foil to impede interference of environment light. The 
reflected light spectrum was continuously scanned through the experimental time, $t$, with a CCD spectrometer using a proper integration time. An integration function was then defined to monitor the total reflected light, $I_{w}$ or the photon counts in the spectrum for a wavelength range, $w$, between 585 and $615 \mathrm{~nm}$, where crystal particles were found to scatter light strongly:

$$
R(t)=\int_{w=585 n m}^{w=615 n m} I_{w}(t)
$$

The relative solution transmittance, $T(t)$ defined as the fraction of incident light that passed through the solution in the monitored wavelength range $w$, was then calculated as $T(t)=R(t) / R(t=0)$ and normalised for each experiment by assuming $100 \%$ light transmittance at time zero, i.e. $T(t=0)=1$ and $0 \%$ transmittance towards the end of the crystallisation, i.e. $T(t=\infty)=0$.

\section{Crystal characterisation}

The mean crystal size and CSDs of Paracetamol powder were determined using an automatic optical system, the Morphologi G3 (Malvern Instruments, Malvern, UK). Optical microscopy offered a more robust CSD method for sizing Paracetamol crystals in comparison with laser diffraction measurements because it offers the possibility of analysing the sample in the dispersed powder form using compressed air at 1.5 barg and avoided sonication process for dispersion of crystals, which has shown to result in the partial dissolution or agglomeration of Paracetamol crystals (results not shown). Paracetamol is very prone to agglomeration, therefore CSD analysis is especially difficult. Nevertheless, the Morphologi G3 system allows the reproducible, automatic analysis of a large number of particles or crystals and the possibility of using different types of filters to manually discard large agglomerates resulting from crystal caking during the sampling and powder drying process.

For CSD analysis using Morphologi G3 $1.5 \mathrm{mg}$ of dry powder were loaded in $20 \mu \mathrm{m}$ thick aluminium foil and dispersed into the clean, glass surface of the microscope using compressed air at 1.5 barg. The G3 software measured the projected area of the crystals/particles and assigned it the equivalent diameter of a circle, $C_{E}$, through a standard operating procedure (SOP). The crystal size computed this way was based on $C_{E}^{3}$, which would only fit laser scattering measurements for perfectly spherical particles. A typical run comprised a sample of 5,000-10,000 crystals to ensure the statistical significance of the acquired CSD. The reproducibility of CDS measurements was found very high upon SOP optimisation (results not shown).

For SEM of the micronized powders, the dried crystals were spread over a surface and dried overnight in He atmosphere. The samples were then coated with a $20 \mathrm{~nm}$ thick gold coating and scanned at $5 \mathrm{Kev}$ in FEI Philips XL30 FEGSEM equipped with an Oxford Instruments INCA EDX system running a $30 \mathrm{~mm}^{2} \mathrm{SiLi}$ thin window pentafet EDX detector. 
The effect of HPMC on the thermal behaviour above ambient of dry Paracetamol powder was recorded using a Perkin-Elmer Pyris 1 scanning differential calorimeter. For that purpose, approximately $5 \mathrm{mg}$ of sample were heated at $10^{\circ} \mathrm{C} / \mathrm{min}$ from 40 to $180{ }^{\circ} \mathrm{C}$ in perforated crimped aluminium pans while being purged with dry nitrogen. The heating and cooling cycles were performed, intercalated by 1 min holding time.

For analysis of the effect of HPMC on crystal system, powder X-ray diffractions in dried powder were acquired at room temperature on a Philips PW1820 diffractometer using Copper-k-alpha radiation (tube operated at $40 \mathrm{kV}, 40 \mathrm{~mA}$ ), a $\theta-\theta$ goniometer, automatic divergence and $0.2 \mathrm{~mm}$ receiving slits, a silicon secondary monochromator, and a scintillation counter. The XRD trace was scanned in $2-\theta$ from $5^{\circ}$ to $60^{\circ}$, at a rate of $0.050^{\circ}$ of $2 \theta$ per 2 seconds. The powder samples were prepared as flat surfaces in aluminium sample holders.

\section{Results and discussion}

\section{Effect of HPMC on the kinetics of Paracetamol crystallisation}

Paracetamol was crystallised in water in a small batch stirred glass vessel containing a reduced HPMC concentration, by quickly dropping the temperature of a slightly undersaturated solution from $70{ }^{\circ} \mathrm{C}$ to $20{ }^{\circ} \mathrm{C}$. Turbidity was on-line monitored during the cooling process using an optical microprobe immersed in the solution, which allowed sensitive detection of the onset of nucleation and quantitative detection of the rate of generation of cloudiness, which is ultimately linked to the generation and growth of crystals. Figure 2 shows the variation of solution temperature and relative transmittance (the lower the transmittance the higher the solution cloudiness) for a selected number of HPMC concentrations. The HPMC concentrations tested in this study were $0.0,0.1,0.3,0.4,0.7$ and $0.8 \% \mathrm{w} / \mathrm{w}$ but only a selection of these is shown in Figure 2 for simplicity in clarity of data presented. The temperature cooling profile was highly reproducible in the whole set of experiments, therefore only one cooling curve is shown in Figure 2. HPMC effectively delayed the onset of nucleation and reduced the rate of generation of cloudiness in the solution as can be confirmed from the plots. With pure Paracetamol, a shower of crystals was detected within 1-2 minutes, and 90\% of the solution cloudiness generated within the following minute, and crystal growth was completed within 5 minutes from the beginning of the cooling process, which compares well with the cooling time of the solution in the vessel. In the presence of HPMC, the time of onset of nucleation extended to up to 9 minutes depending on the HPMC concentration, so well beyond the cooling time of the solution in the vessel. This ultimately means that the crystallisation in the presence of HPMC occurs entirely under full isothermal conditions, at which the solution supersaturation is at its maximum and therefore a shower of crystals beneficial for the production of micronized powders is expected. The ability of running solution crystallisations in full isothermal conditions as shown in 
this manuscript is novel and might find broad applications in the direct crystallisation of micronized APIs.

The turbidity profiles in Figure 2 did not allow the full deconvolution of crystal nucleation and crystal growth rates, however some quantitative information was extracted in order to quantify the effect of polymer additive in the overall batch cooling crystallisation kinetics. For that purpose, an arbitrary induction time, $t_{i n d}$ and crystal growth completion time, $t_{G}$ were defined corresponding to the time required to give a $20 \%$ and $80 \%$ decrease, respectively, in solution transmittance. For this analysis the whole range of HPMC concentrations tested was considered. Equally, a maximum generated cloudiness rate, $R_{c}$, was calculated from the initial gradient of relative solution absorbance versus cooling time, which is summarised in Figure 3a. During the initial stage of crystal growth, when submicron nuclei grow to a size to be optically detected and therefore the concentration of crystals is proportional to the absorbance of the solution, the increase in solution absorbance (defined as $-\log _{10}$ of transmittance) is essentially related to the increase in the concentration of particles or the formation of new crystals, therefore $R_{c}$ should capture the relative effect of polymer additive concentration on kinetics of crystal nucleation. Figure 3a showed that $t_{\text {ind }}$ increased by up to 5.4-fold and $t_{G}$ by up to 9.5-fold upon the addition of HPCM to the solution, with the maximum effect being observed at the highest HPMC concentration tested of $0.8 \% \mathrm{w} / \mathrm{w}$. Equally, $R_{c}$ decreased up to $73.4 \%$ with increasing HPMC concentration, being the inhibitory effect more noticeable at low HPMC concentrations. This represents a major effect of polymer additive in respect to the overall kinetics of crystallisation, from where a major reduction in crystal size could be expected.

\section{Effect of HPMC on mean crystal size and CSD}

A common motivation for controlling crystallisation processes in pharmaceutical industries is the production of uniform particle sizes with a given mean crystal size. Therefore, the Paracetamol powder produced in the presence of different HPMC concentrations has been characterised in respect to particle size using the Morphologi G3 system, which applies compressed air for dispersing dry powders on a glass microscope slide and advanced imaged analysis to determine a circle equivalent diameter, $C_{E}$ of the crystals. This allowed confirming that the presence of HPMC in the initial crystallisation solution resulted in a significant reduction in the mean crystal size, $D_{50}$ and an improvement in CSD as shown in Figures $4 \mathrm{a}$ and $4 \mathrm{~b}$. With pure Paracetamol the $D_{50}$ obtained was $39.6 \mu \mathrm{m}$. This was per si considerably smaller than the mean sizes reported by other authors for batch cooling crystallisation from solution. For example, Chew et al. ${ }^{17}$ and Fujiwara et $a l . .^{5}$ reported a $D_{50}$ in the range of $100-250 \mu \mathrm{m}$ for batch crystallisers, and Zarkadas \& Sirkar ${ }^{18}$ produced crystal sizes with $50-150 \mu \mathrm{m}$ in a continuous hollow fibre device. The smaller crystal 
sizes obtained in this study are related to the large surface area-to-volume ratio of the glass vessel used in the crystallisation runs, which delivered high cooling rates for high supersaturation and nucleation rates that cannot be mimicked in larger crystallisers. Nevertheless, the CSD in Figure 4a was broad as can be seen from be great deviation of the values represented by percentile $10, D_{10}=$ $15.5 \mu \mathrm{m}$ and percentile $90, D_{90}=62.1 \mu \mathrm{m}$. This could be associated with the non-isothermal conditions in the crystallisation vessel at the time of onset of solution cloudiness. The addition of $0.1 \% \mathrm{w} / \mathrm{w}$ of HPMC resulted in $D_{50}$ decreasing from 39.6 to $15.4 \mu \mathrm{m}$ and the volume-based CSD becoming remarkably sharper, with $D_{10}=8.3 \mu \mathrm{m}$ and $D_{90}=22.6 \mu \mathrm{m}$. The frequency-based CSD in Figure $4 \mathrm{~b}$ confirmed a larger number of fines in the dried powder crystallised in the presence of $0.1 \% \mathrm{w} / \mathrm{w}$ HPMC, and smaller number of large crystals. The dashed vertical line represents the smallest crystal size that could be detected in with the used setup with Morphologi G3. That significant decrease in the mean crystal size was confirmed by SEM images in Figures $4 \mathrm{c}$ and $4 \mathrm{~d}$ (note the different scales in the SEM microphotographs).

The effect of varying initial concentrations of HPMC on particle size distribution in respect to percentile $10, D_{10}$, percentile $50, D_{50}$ and percentile $90, D_{90}$ is fully summarised in Figure $3 \mathrm{~b}$. The use of HPMC concentrations above $0.1 \% \mathrm{w} / \mathrm{w}$ resulted in no further improvement in particle size reduction or CSD, and it appeared that the presence of a very small concentration of HPMC in the solution was sufficient to provide a good level of particle size control.

Another aspect that contributed to smaller crystal sizes was the fact that Paracetamol solubility decreases very quickly with HPMC content, as shown in supplementary data (Figure S1). For a $0.1 \%$ mass ratio of HPMC to Paracetamol (corresponding to approximately $0.03 \% \mathrm{w} / \mathrm{w}$ of HPMC in respect to mass of solution) the solubility measured at $30{ }^{\circ} \mathrm{C}$ dropped by $40 \%$. This means that in the presence of HPMC the crystallisation is isothermal but also supersaturation is higher than for a pure Paracetamol system. This should in theory return higher crystallisation yields, and in respect to particle size control this represents enhanced supersaturation in the presence of HPMC which favours production of smaller crystals and more fines.

Overall and based on the turbimetry data, it is not possible to identify the main mechanic leading to a significant reduction in crystal size and improvement in CSD in the presence of HPMC, but it appears linked to a higher supersaturation and tighter supersaturation control resulting from the delay of onset of nucleation, higher number of fines and reduction in Paracetamol solubility.

\section{Effect of HPMC on crystal morphology}

A number of recent studies mainly performed at static conditions have linked the use of organic polymer additives with crystal polymorphism as reviewed in the Introduction section. A given crystal form can be inhibited upon the presence of polymer or "impurities", therefore polymer 
additives can induce selective production of a given polymorph. ${ }^{6-9}$ Equally, other studies have reported a modification of crystal habit upon the addition of polymer additives. ${ }^{8,9}$ Mainly with isentropic crystals like Paracetamol, ${ }^{20}$ polymers can present higher selectivity to bind given faces a crystal, so selectively inhibiting the growth on a given direction. In order to test for possible effects of HPMC additive on crystal structure, Paracetamol powders were characterised regarding their thermal behaviours and X-ray diffraction patterns. Optical and electronic microscopic analysis of the crystals showed that crystals shape changed to an elongated-prismatic shape upon the addition of polymer. The DSC and XRD data in Figures 5 and $5 \mathrm{~b}$, respectively, confirmed that the crystalline product obtained with pure Paracetamol and $0.1 \% \mathrm{w} / \mathrm{w}$ of HPMC were in fact from the same crystal form I, i.e. monoclinic Paracetamol. The experimental XRD patterns of the two Paracetamol samples crystallised with $0 \%$ and $0.1 \%$ w/w of HPMC compared in Figure 4 agreed well with XRD data of e.g. Martino et al. ${ }^{21}$ and Nichols and Frampton ${ }^{22}$ by showing peaks unique to the monoclinic form I such as $2 \theta=12.025,15.425$ and 26.475. Also, the melting point (endothermic) at $164{ }^{\circ} \mathrm{C}$ and two crystallisation (exothermic) points at $88-89^{\circ} \mathrm{C}$ and $135-139{ }^{\circ} \mathrm{C}$ shown by DSC analysis in Figure 5b also confirmed that the two samples were of the same crystal form. This confirmed that the detected difference in kinetics of crystallisation is not due to the production of a different polymorph.

Detailed SEM analysis to all Paracetamol samples revealed a side effect with the use of HPMC concentrations of $0.3 \% \mathrm{w} / \mathrm{w}$ and above, with extended agglomeration and a large number of cavities or holes on the crystal surface. Figure 6 shows cavities up to 100-200 nanometers in diameter that become more prominent as more HPMC was added to the solution. The reason behind the formation of these cavities is unknown, however it is suspect to be linked to the intermolecular interactions of HPMC and Paracetamol, with HPMC adsorbing the crystal surface as suggested by Thompson et al. ${ }^{23}$ for Metacetamol (a small molecule with -OH groups). Using AFM and SEM, they have shown surface features in pure Paracetamol crystals between 1 and $20 \mathrm{~nm}$, that changed to $15 \mathrm{~nm}$ steps interspersed with holes in the presence of Metacetamol.

\section{Effect of HPMC on final product yield}

The product yield calculated in terms of dried powder weight was found independent of the HPMC concentration used, and in average $59.5 \pm 1.62 \%$ as shown in Figure 3c. This was slightly lower than the theoretical yield expected from pure Paracetamol $(74.1 \%)$ based on the solubility data of Granberg\&Rasmuson. ${ }^{19}$ Note that the addition of HPMC to the solution can only increase the theoretical yield up to a maximum of $3.6 \%$ assuming all HPMC added to the solution ends up in the dried powder. Based on the solubility data shown in Figure S1 the addition of HPMC to the crystallisation solution should have resulted in higher yield than for pure Paracetamol, as HPMC 
was found to decrease significantly the solubility of Paracetamol in water. This might be related to the dissolution of some material during the sample washing or to surface poisoning. Femi-Oyewo \& Spring ${ }^{10}$ reported $91 \%$ reduction on yield of Paracetamol crystallised in the presence of HPMC under static conditions, which was shown to be related to the irreversible adsorption of polymer of the surface of the crystals. This data herein reported shows that under well mixed conditions the adsorption of polymer to the surface of the crystals is reversible however it cannot entirely overcome surface poisoning that leads to the terminal size of the crystal.

\section{Remarks on the effect of HPMC on kinetics of Paracetamol crystallisation}

The mechanics for delaying the onset of nucleation and reducing the rate of solution cloudiness in cooling crystallisation of Paracetamol crystals by HPMC is still unknown, however recent studies have proposed different hypothesis spanning from inhibition of heterogeneous nucleation to increased mass transfer resistances both related to the hydrogen bounding between the polymer and Paracetamol molecules. This section offers an overview to those important mechanisms that support the possible impact of HPMC on the nucleation and/or growth of Paracetamol crystals.

Heterogeneous nucleation is thermodynamically more favourable and is known to be related to the presence of interfaces, such as crystalliser's wall, impeller or air-waste free surface. Zarkadas $\&$ Sirkar ${ }^{18}$ have found that a combination of homogeneous and heterogeneous nucleation is possible in a cooling crystallisation process of Paracetamol in water at high supersaturation ratios, i.e. $S=3$ and above, which was the case in this present study. In order to validate this hypothesis a further set of experiments was carried out in a combination of experimental conditions that were recognised to promote heterogeneous nucleation, such is the case of stirring, glass wall material and air-water interface, as summarised in Table 1. For each crystallisation run, the time required to detect the onset of solution cloudiness was noted (in these set of experiments the induction time was determined using naked eye). It was observed that the addition of $0.1 \% \mathrm{w} / \mathrm{w}$ HPMC has similar effect in respect to the delay on induction time to the removal of free surface during the crystallisation vessels, resulting in delayed induction time from 4.0 to 9-10 min. This is likely to be associated with the fact that HPMC reduced the frequency of collisions between the solute particles, which, in turn, led to the delay of nucleation. The rate for primary nucleation is linearly proportional to the diffusion coefficient ${ }^{4}$ of Paracetamol, having a molecular weight of $151.2 \mathrm{~g} / \mathrm{mol}$. When the diffusion is sufficiently rapid the nucleation is not diffusion controlled. This is normally the case at high values of $S$, and explains the very short induction time obtained with pure Paracetamol (run 1 in Table 1). Nevertheless, with the presence of HPMC in the crystallisation solution the nucleation became increasingly limited as more polymer additive is added to the solution. This was explained by Zarkadas\&Sirkar ${ }^{18}$ and Tian et al. ${ }^{24}$ based on inter-molecular 
hydrogen bonding between Paracetamol and the polymer additive. HPMC has both a hydrogen bonding donor and acceptor groups on its ring structure (Figure S2), therefore showing a high Hbonding ability and network association with drug molecules as concluded from FT-IR results of Tian et al. ${ }^{24}$ Similar conclusion was obtained by Sahoo et al. ${ }^{25}$ using Raman spectroscopy. The last have identified intra-molecular hydrogen bounding between HPMC and Ciprofloxacin, resulting from hydroxyl $\mathrm{OH}$ - groups present in both polymer additive and drug molecules. Raghavan et al. ${ }^{15}$ has also reported strong effect of cellulose polymers on morphology of hydrocortisone acetate. This means HPMC effectively can work as a "molecular transporter" therefore limiting both the diffusivity of drug molecules and reducing the impact probability for the formation of nuclei through collisions.

The production of smaller crystals and the reported morphological changes could also be explained based on several growth-inhibition hypothesis, such as $i$ ) mass transfer limitations, ii) impurities deposition, $\left.{ }^{25} i i i\right)$ polymer adsorption on the surface of the crystals, or $i v$ ) a combination of those. During growth some impurities can adsorb to the surface of the crystals "blocking" the active growth sites (sometimes only blocking growth of a given face of the crystal) or entirely suppresses crystal growth. ${ }^{26}$ HPMC was previously shown capable of adsorbing the surface of Paracetamol crystals. ${ }^{9}$ This has been linked to a reduction in the growth rate of steps when compared to pure Paracetamol. Using AFM and SEM, Thompson et al. ${ }^{23}$ showed that metacetamol changed the growth steps of Paracetamol and disrupting the crystal lattice by adsorbing to the surface and inducing the formation of defects or cavities in the crystal surface.

A parallel observation from Table 1 is the relevant effect of mixing and interfaces on the crystallisation of Paracetamol in respect to induction time in the presence of $0.10 \% \mathrm{w} / \mathrm{w}$ HPMC. Although it is difficult to separate the effect of interfaces from that of mixing, it was observed that induction time approximately doubled in the absence of headspace in the crystallisation vessel. Interfaces are known for creating temperature gradients therefore being preferential zones for nucleation. On the other hand, the removal of agitation resulted in further increased induction times (rows 6 and 7), however this is believed to be related to the lower cooling rate in unstirred vessel.

\section{Conclusions}

Batch cooling crystallisation experiments of Paracetamol in water in the presence $0.1-0.8 \% \mathrm{w} / \mathrm{w}$ of HPMC revealed the ability of the natural polymer additive modifying the overall kinetics of crystallisation by slowing the rate of generation of cloudiness in the solution and delaying the onset of nucleation beyond the cooling time of a crystalliser. This allowed direct production of micronized Paracetamol crystals from solution, with a $D_{50}$ in the range of $15-20 \mu \mathrm{m}$ and sharp CDS, which was linked to the isothermal crystallisation conditions in the presence of HPMC and 
reduction of Paracetamol solubility in the presence of polymer. The observed habit change induced by the presence of HPMC suggested the polymer acted by inhibiting growth on specific surfaces of the crystals, as previously reported for other crystallisation systems. The high density of cavities observed on the surface of the crystals for higher HPMC concentrations strongly suggests that HPMC adsorbed to surface of the crystals disrupting the hydrogen bounding network. This technique can potentially be used for enhancing direct crystallisation of other micronized drugs for pulmonary drug delivery.

\section{Acknowledgment}

The authors are grateful to Dr Jeremy Skepper from Multi Imaging Centre, Cambridge, for help with SEM; Dr Eric Rees and Dr Joanna Stasiak for help with XRD; Zlatko Saracevic for help with DSC analysis, and Henrietta Adomako at Loughborough University for assistance with Paracetamol solubility experiments. NMR is grateful to Fundacao para a Ciencia e Tecnologia, FCT (SFRH/BPD/26904/2005) and European Commission Marie Curie IEF programme (project 221768) for financial support.

\section{Supporting Information Available}

Solubility data for Paracetamol in the presence of different mass ratios of HPMC, and chemical structure of Paracetamol and HPMC molecules. This information is available free of charge via the Internet at http://pubs.acs.org/.

\section{References}

(1) Pulmonary Drug Delivery Systems: Technologies and Global Markets; Rockville, MD, USA, 2012; p. 222.

(2) Steckel, H. International Journal of Pharmaceutics 2003, 258, 65-75.

(3) Martin, G. P.; Zeng, X. M. Pharmaceutical composition for pulmonary delivery. WO/2001/058425, 2001.

(4) Garside, J.; Mersmann, A.; Nyvlt, J. Measurement of crystal growth and nucleation rates; 2nd editio.; IChemE: Rugby, UK, 2002.

(5) Fujiwara, M.; Nagy, Z. K.; Chew, J. W.; Braatz, R. D. Journal of Process Control 2005, 15, 493-504.

(6) Grzesiak, A. L.; Matzger, A. J. Crystal growth \& design 2008, 8, 347-350.

(7) Price, C. P.; Grzesiak, A. L.; Matzger, A. J. Journal of the American Chemical Society 2005, 127, 5512-7.

(8) Lang, M.; Grzesiak, A. L.; Matzger, A. J. Journal of the American Chemical Society 2002, 124, 14834-5.

(9) Capes, J. S.; Cameron, R. E. CrystEngComm 2007, 9, 84.

(10) Femi-Oyewo, M. N.; Spring, M. S. International Journal of Pharmaceutics 1994, 112, 17-28. 
(11) Pawar, A.; Paradkar, A. R.; Kadam, S. S.; Mahadik, K. R. Indian Journal of Pharmaceutical Sciences 2007, 69, 658-664.

(12) Xie, S.; Poornachary, S. K.; Chow, P. S.; Tan, R. B. H. Crystal Growth \& Design 2010, 10, 3363-3371.

(13) Davey, R. J.; Blagden, N.; Potts, G. D.; Docherty, R. Journal of American Chemical Society 1997, 7, 1767-1772.

(14) Gu, C.-H.; Chatterjee, K.; Young, V.; Grant, D. J. Journal of Crystal Growth 2002, 235, 471481.

(15) Raghavan, S. L.; Trividic, a; Davis, a F.; Hadgraft, J. International journal of pharmaceutics 2001, 212, 213-21.

(16) Weiner, M. L.; Kotkoskie, L. A. Excipient Toxicity and Safety (Drugs and the Pharmaceutical Sciences); Marcel Dekker: New York, USA, 2000.

(17) Chew, C. M.; Ristic, R. I.; Dennehy, R. D.; De Yoreo, J. J. Crystal Growth \& Design 2004, 4, $1045-1052$.

(18) Zarkadas, D. M.; Sirkar, K. K. Industrial \& Engineering Chemistry Research 2007, 46, $2928-$ 2935.

(19) Granberg, R. A.; Rasmuson, Å. C. Journal of Chemical \& Engineering Data 2000, 45, 478483.

(20) Heng, J. Y. Y.; Williams, D. R. Langmuir : the ACS journal of surfaces and colloids 2006, 22, 6905-9.

(21) Martino, P. Di; Guyot-Hermann, A.-M.; Conflant, P.; Drache, M.; Guyot, J.-C. International Journal of Pharmaceutics 1996, 128, 1-8.

(22) Nichols, G.; Frampton, C. S. Journal of pharmaceutical sciences 1998, 87, 684-93.

(23) Thompson, C.; Davies, M. C.; Roberts, C. J.; Tendler, S. J. B.; Wilkinson, M. J. International journal of pharmaceutics 2004, 280, 137-150.

(24) Tian, F.; Baldursdottir, S.; Rantanen, J. Molecular Phamaceuticals 2009, 6, 202-210.

(25) Sahoo, S.; Chakraborti, C. K.; Behera, P. K. International Journal of Applied Pharmaceuticals 2012, 4.

(26) Myerson, A. Handbook of Industrial Crystallization; Myerson, A., Ed.; Butterworth Heinemann: Boston, USA, 2001.

(27) Mullin, J.W. Industrial Crystallization. Butterworth- Heinemann: London, 1993. 
Tables

Table 1. Induction times detected by naked eye, $t_{\text {ind }}$, for batch cooling crystallisation of Paracetamol with HPMC under different nucleation conditions

\begin{tabular}{cccccc}
\hline Run & $\begin{array}{c}\text { Wall } \\
\text { material }\end{array}$ & $\begin{array}{c}\text { Water-air } \\
\text { interface }\end{array}$ & Mixing & $\begin{array}{c}\text { HPMC concentration } \\
(\mathrm{w} / \mathrm{w})\end{array}$ & $\begin{array}{c}t_{\text {ind }}{ }^{\prime} \\
(\mathrm{min})\end{array}$ \\
\hline 1 & Glass & Yes & Yes & $0.00 \%$ & 1.7 \\
2 & Glass & Yes & Yes & $0.10 \%$ & 4.0 \\
3 & Glass & No & Yes & $0.10 \%$ & 9.5 \\
4 & LLDPE & Yes & Yes & $0.10 \%$ & 4.4 \\
5 & LLDPE & No & Yes & $0.10 \%$ & 10.5 \\
6 & LLDPE & Yes & No & $0.10 \%$ & 15.2 \\
7 & LLDPE & No & No & $0.10 \%$ & 20.5
\end{tabular}

20

21

22

23

24

25

26

27

28

29

30

31

32

33

34

35

36

37

38

39

40

41

42

43

44

45

46

47

48

49

50

51

52

53

54

55

56

57

58

59

60 
Figures list

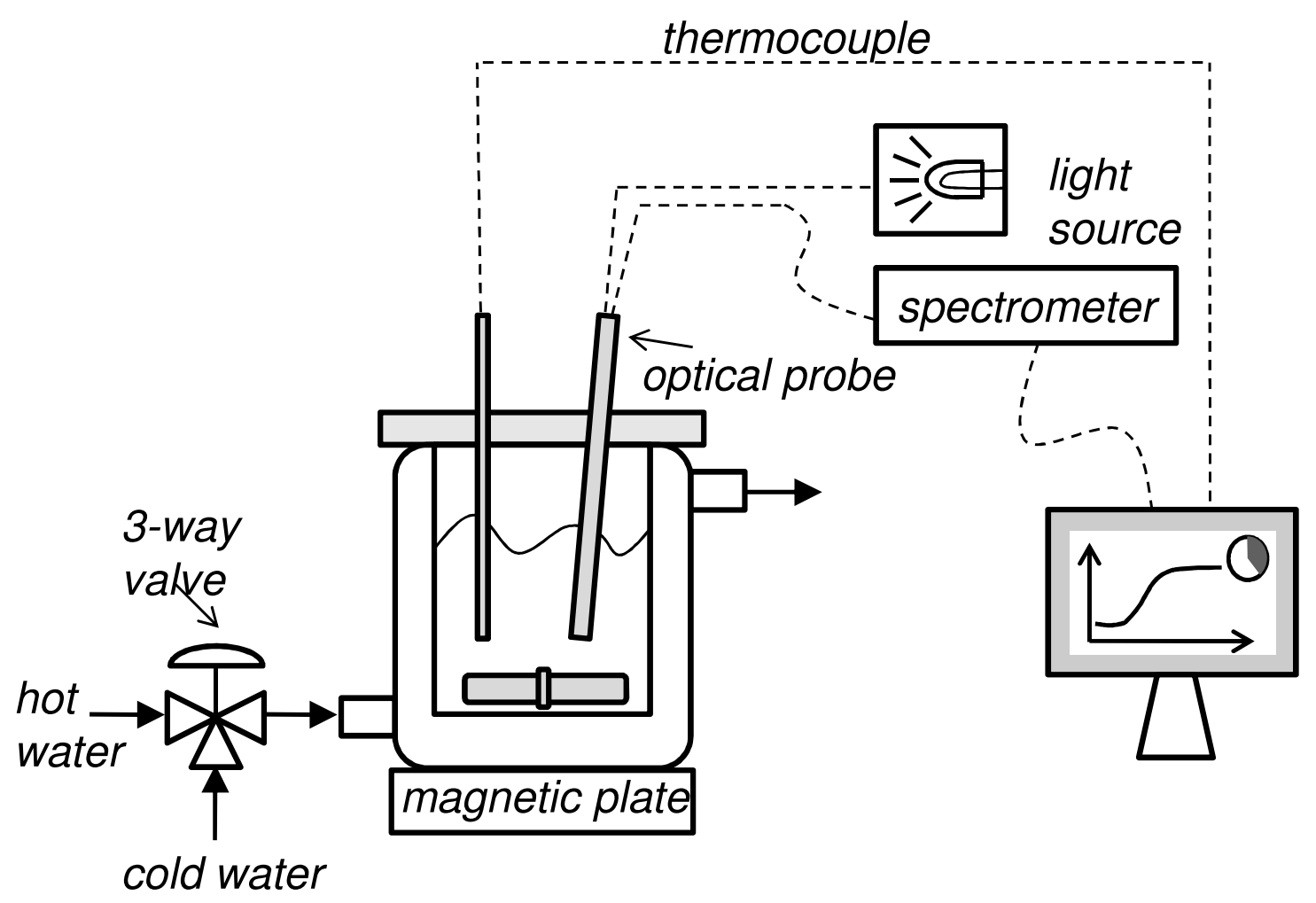

Figure 1. Experimental setup used in the batch cooling crystallisation experiments. 

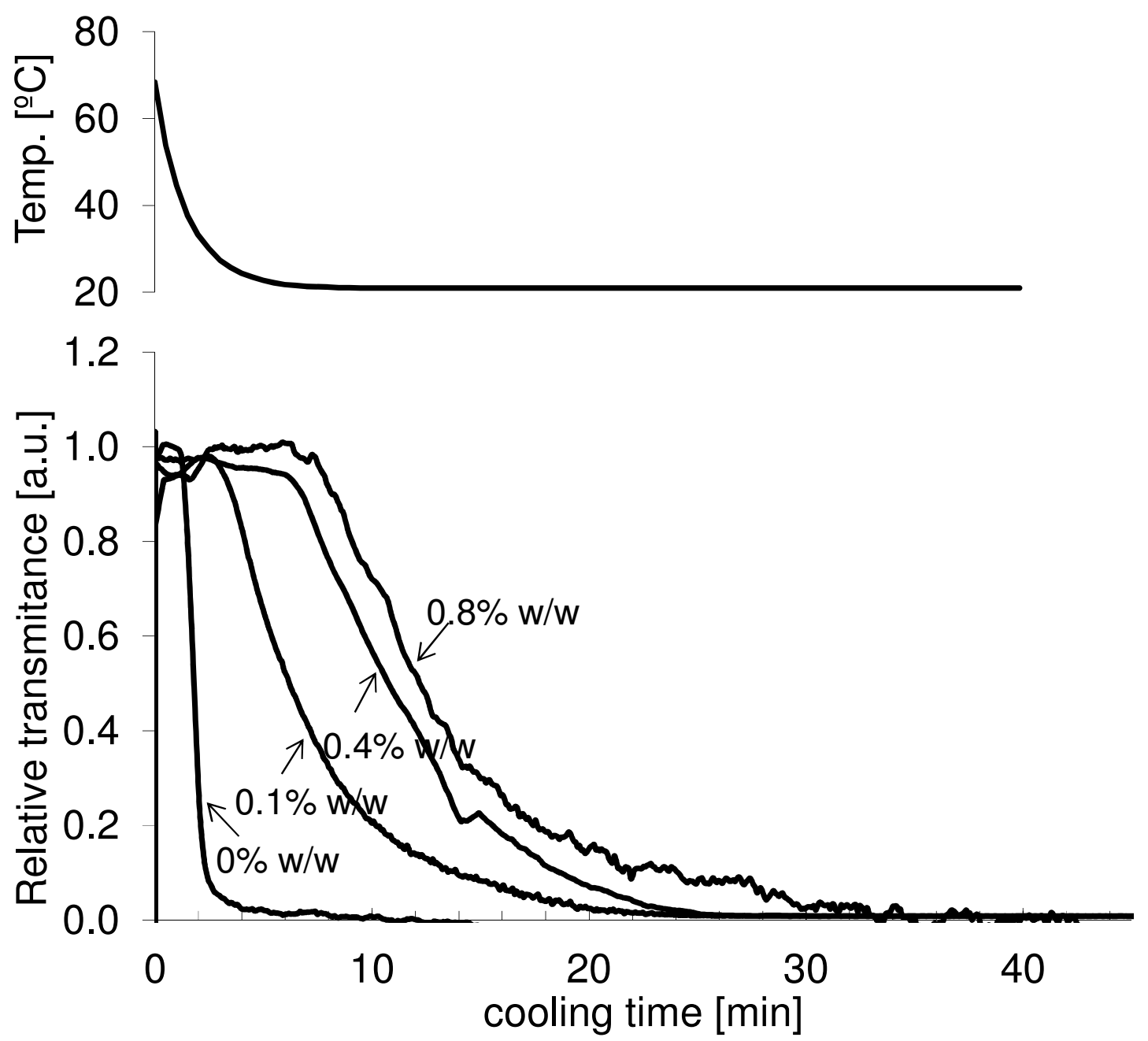

Figure 2. Real-time profiles temperature and relative solution transmittance (or turbidity) in the vessel during batch cooling crystallisation. For clarity, only HPMC concentrations of $0 \%, 0.1 \%$, $0.4 \%$ and $0.8 \%$ w/w were shown. 


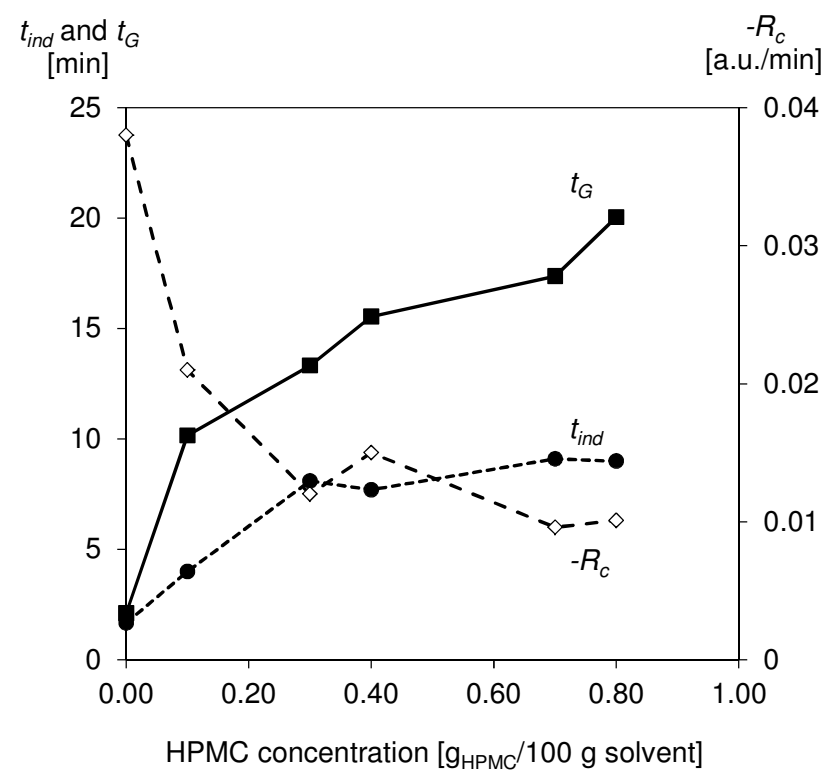

(a)

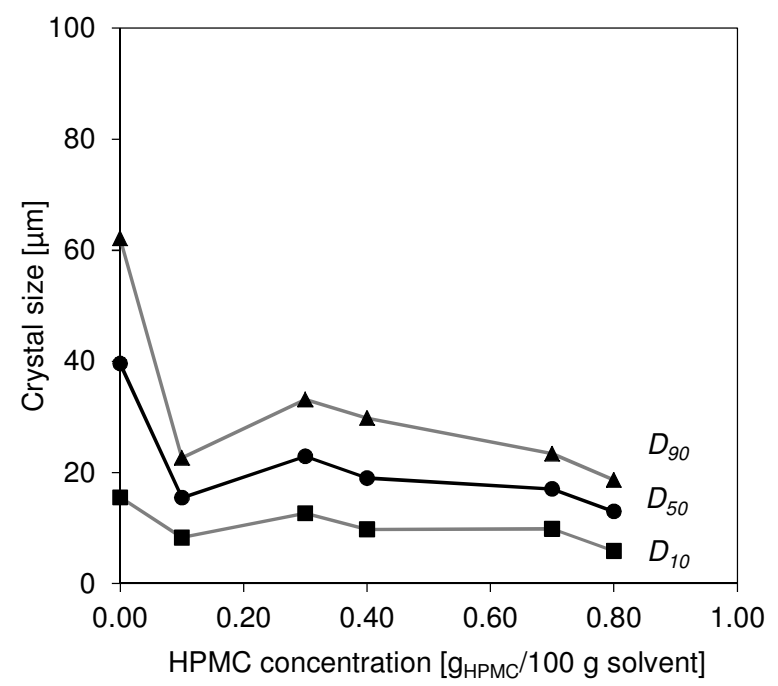

(b)

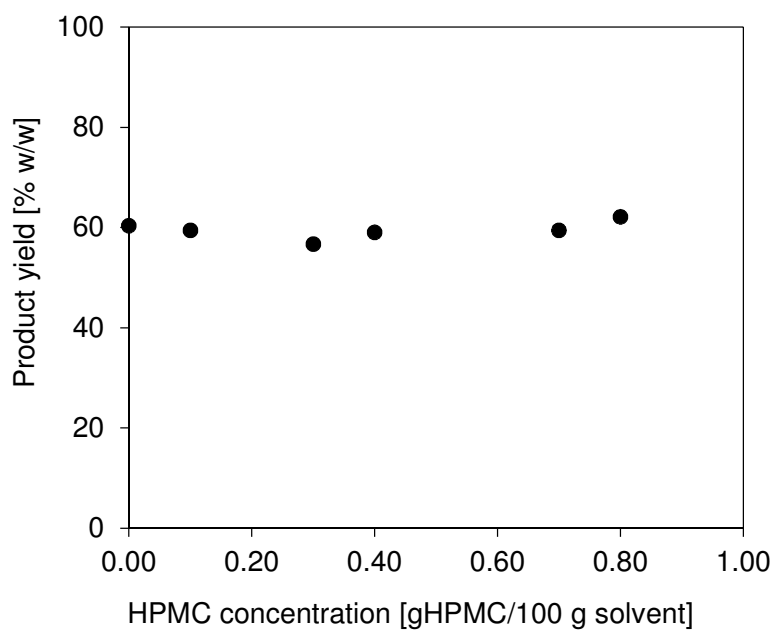

(c) 
Figure 3. Effect of initial HPMC concentration on (a) induction time, $t_{\text {ind }}$ crystal growth time, $t_{G}$ and maximum rate of generation solution cloudiness, $R_{c}$ as determined from turbidity plots in Figure 2; (b) crystal size distribution of dried crystals, and (c) final product yield, for 45 min of cooling. For more details calculating $t_{i n d}, t_{G}$ and $R_{c}$ in (a) see text. The product yield expected from the solubility data of Granberg\&Rasmuson ${ }^{19}$ was $73.5 \% \mathrm{w} / \mathrm{w}$, which is plotted in (c) as a guideline.

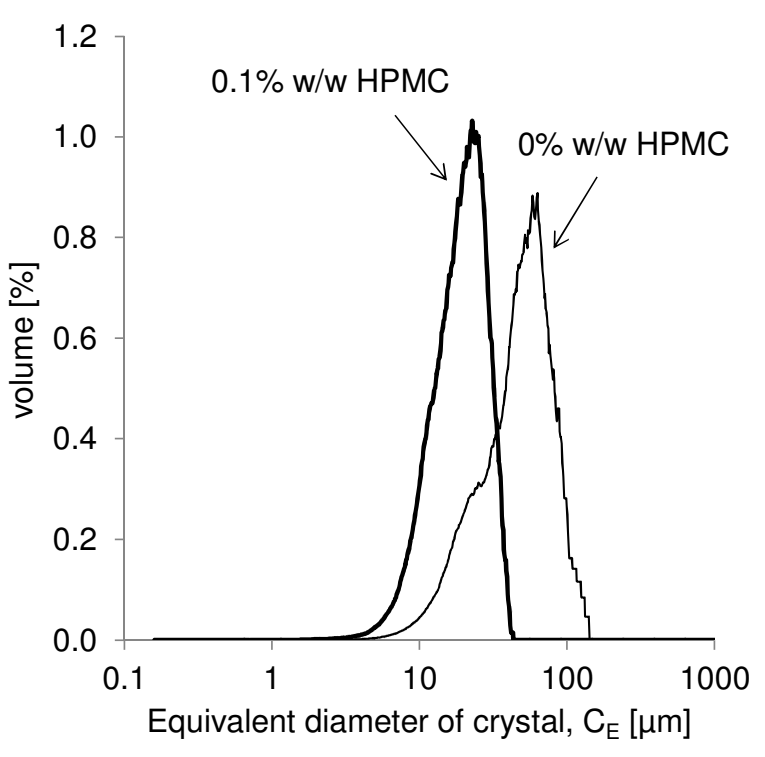

(a)

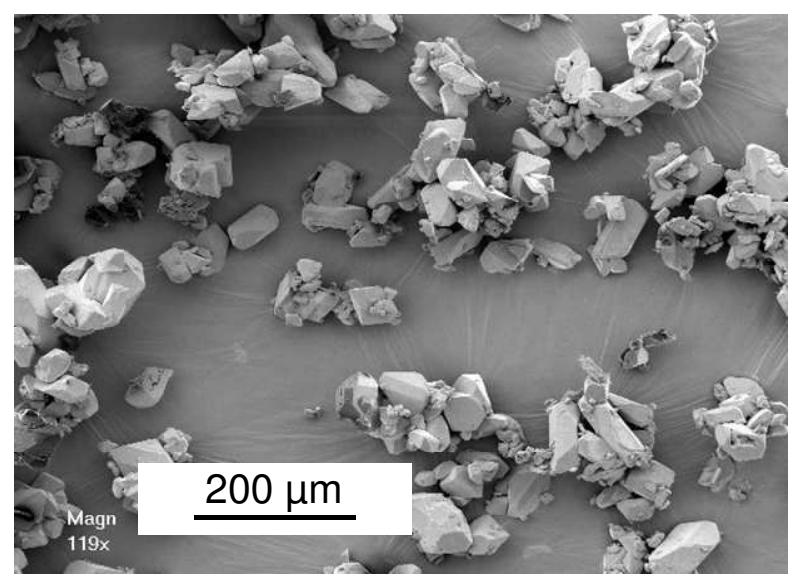

(c)

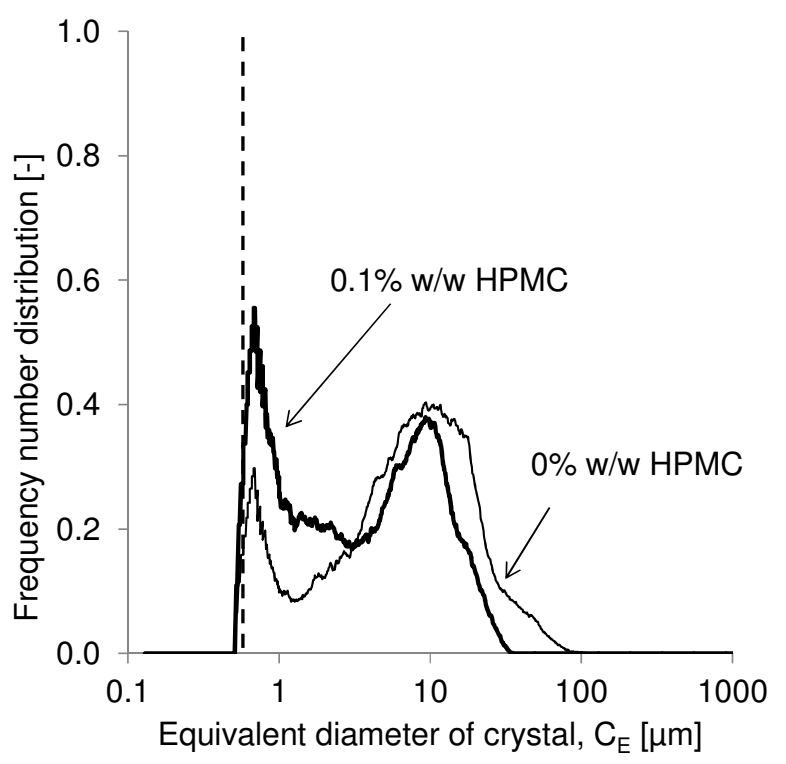

(b)

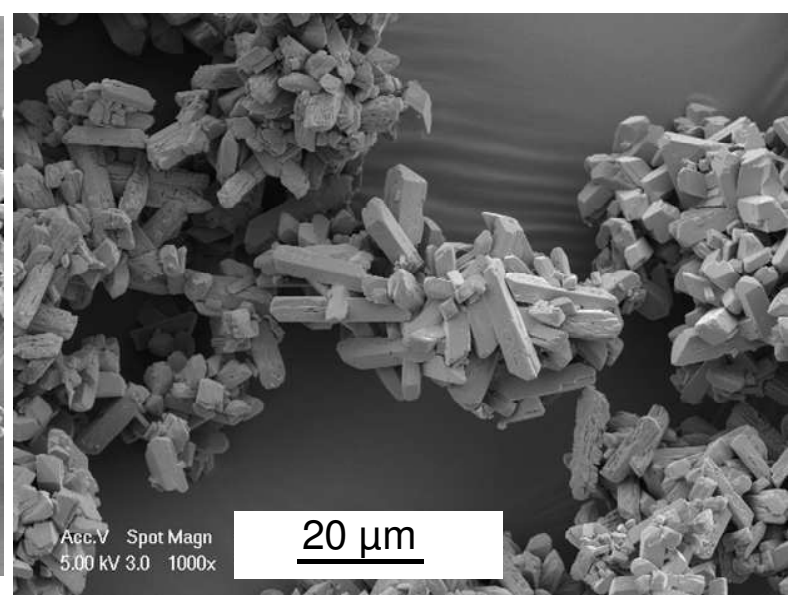

(d)

Figure 4. Crystal size distribution for Paracetamol crystallised in $0 \% \mathrm{w} / \mathrm{w}$ and $0.1 \% \mathrm{w} / \mathrm{w}$ of HPMC based on (a) volume and (b) frequency. SEM images of Paracetamol samples crystallised in (c) $0 \%$ w/w HPMC and (d) $0.1 \%$ w/w HPMC. Note the different scale bars used in (c) and (d). 


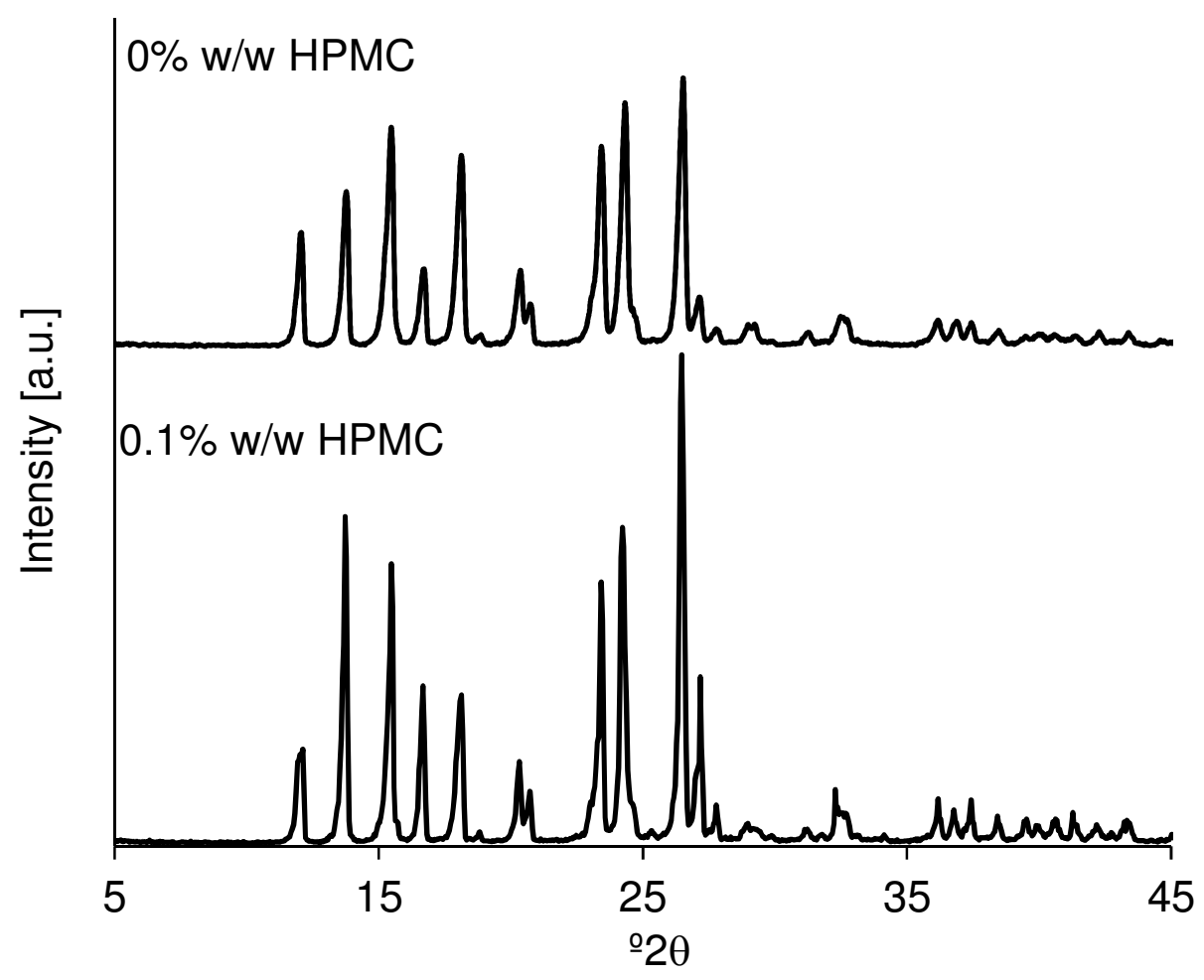

(a)

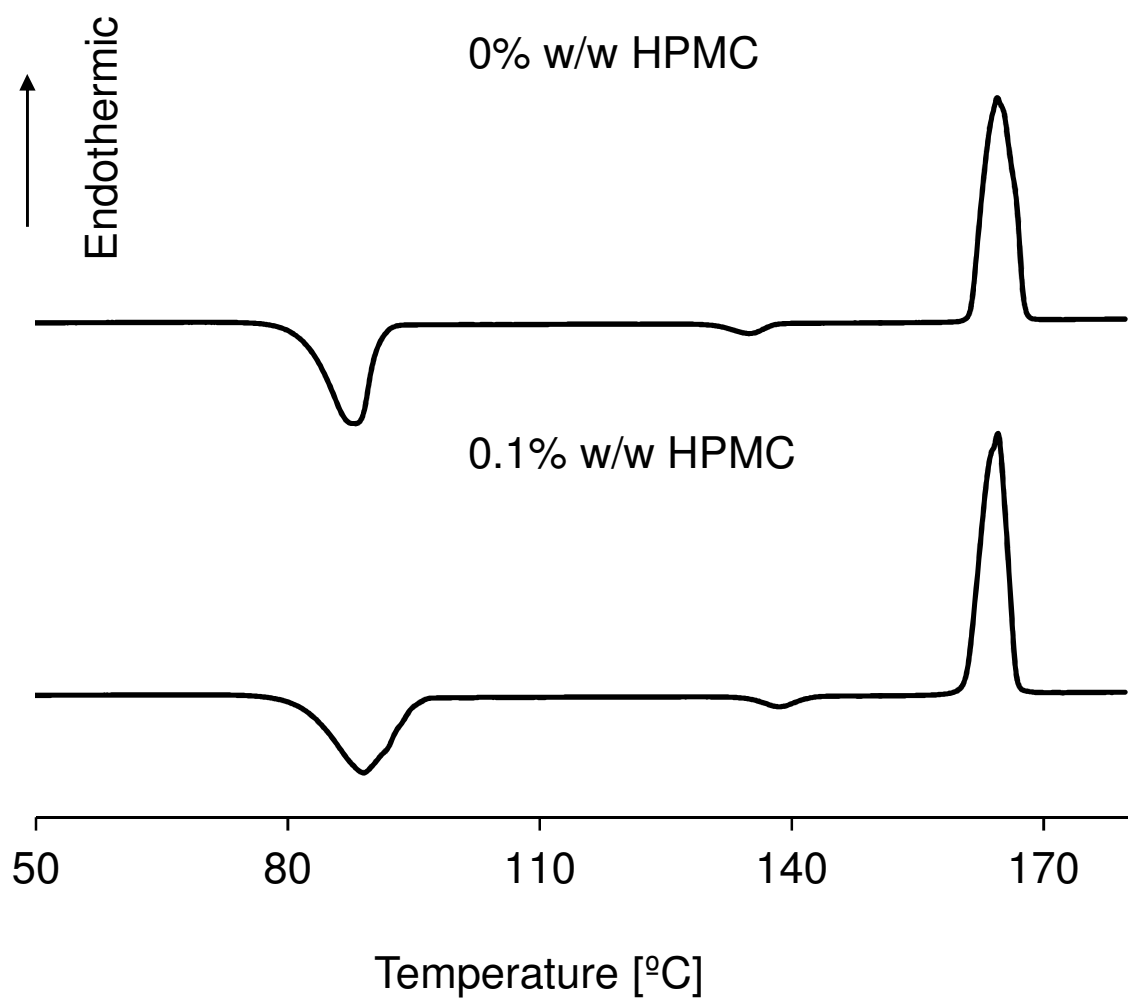

(b)

Figure 5. (a) XRD patterns and (b) DSCs for Paracetamol crystallised in $0 \% \mathrm{w} / \mathrm{w}$ and $0.1 \% \mathrm{w} / \mathrm{w}$ of HPMC. 


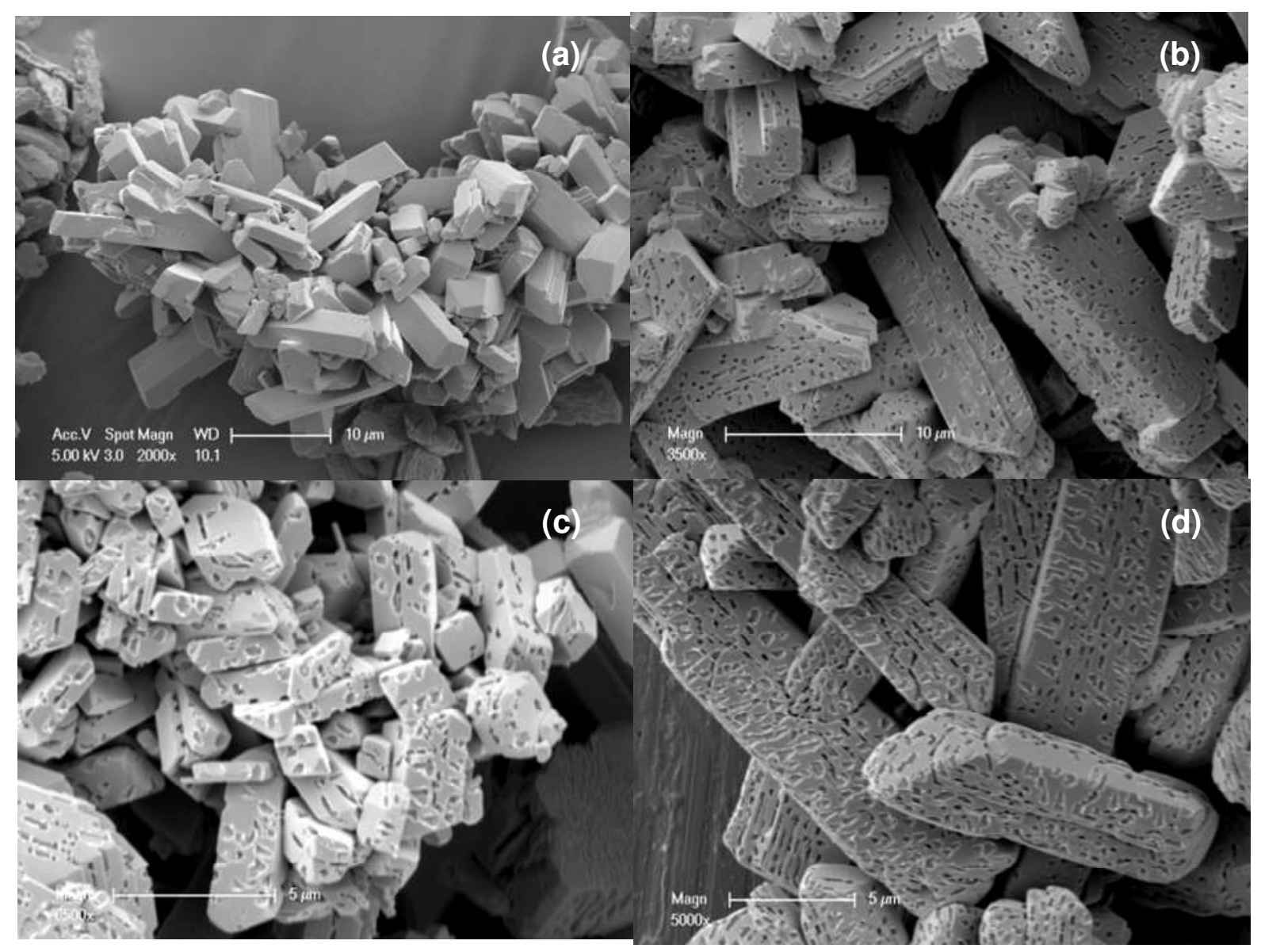

Figure 6. SEM images of Paracetamol crystallised in the presence of (a) $0.1 \% \mathrm{w} / \mathrm{w}$, (b) $0.3 \% \mathrm{w} / \mathrm{w}$, (c) $0.7 \% \mathrm{w} / \mathrm{w}$ and (d) $0.8 \% \mathrm{w} / \mathrm{w}$ of HPMC showing microporosity in crystal's surface. 


\title{
For Table of Contents Only
}

\section{HPMC as a novel tool for isothermal solution crystallisation of micronized Paracetamol}

\author{
Nuno M Reis ${ }^{\mathrm{a}, \mathrm{b}, \vec{z}}$, Zizheng K Liu ${ }^{\mathrm{a}}$, Cassilda M Reis ${ }^{\mathrm{a}}$, and Malcolm R Mackley ${ }^{\mathrm{a}}$ \\ ${ }^{a}$ Department of Chemical Engineering and Biotechnology, University of Cambridge, New Museums \\ Site, Pembroke Street, Cambridge CB2 3RA, UK \\ ${ }^{b}$ Department of Chemical Engineering, Loughborough University, Loughborough LE11 3TU, UK
}

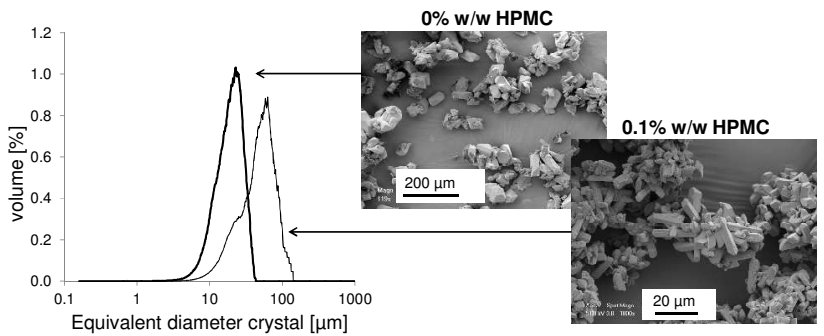

Micronized Paracetamol crystals were directly produced from solution crystallisation in water by quickly dropping the temperature in the presence of $0.1-0.8 \% \mathrm{w} / \mathrm{w}$ of HPMC. The polymer reduced the solubility of Paracetamol and delayed the unset of nucleation beyond the cooling time of the vessel, consequently allowing to produce fine crystals with sharp CSDs.

\footnotetext{
¥ Corresponding author: Phone +44 (0) 1509222 505; Fax +44 (0) 1509223 923; e-mail n.m.reis@ @ lboro.ac.uk
} 